\title{
Spanning trees in subgraphs of lattices
}

\author{
Fan Chung* \\ University of California, San Diego \\ La Jolla, 92093-0112
}

\section{Introduction}

In a graph $G$, for a subset $S$ of the vertex set, the induced subgraph determined by $S$ has edge set consisting of all edges of $G$ with both endpoints in $S$. The (edge) boundary, denoted by $\partial S$ consists of all edges containing one endpoint in $S$ and one endpoint not in $S$.

We consider the combinatorial Laplacian of a graph and an induced subgraph of a graph. Using the classical matrix-tree theorem [8], the number of spanning trees of a graph is proportional to the product of nonzero eigenvalues of the combinatorial Laplacian. We will introduce the zeta function of a graph and derive its relation to the heat kernel and the number of spanning trees of a graph.

In the second part of the paper, we will focus on the special case which involves induced subgraphs of a lattice graph. We will show that for a connected induced subgraph $S$ of a 2-dimensional lattice graph, the number of spanning trees $\tau(S)$ satisfies

$$
c e^{c_{1}|S|-c_{2}|\partial S|} \leq \tau(S) \leq c^{\prime} e^{c_{1}|S|+c_{3}|\partial S|^{2} /|S|}
$$

where the constants $c_{1}, c_{2}$ and $c_{3}$ are universal contants depending only on the host graph but independent of $S$.

This can be viewed as a discrete analog of the classical results of H. Weyl [10] and McKean and Singer [9]. This paper is organized as follows. In Section 2, we

* Research supported in part by NSF Grant No. DMS 98-01446 
state the relationship of the combinatorial Laplacian and the number of spanning trees. In Section 3, we consider the heat kernel and the zeta function of a graph. In Section 4, we focus on the heat kernel of a lattice graph. In Section 5, we prove the main theorem by using the tools defined in preceding sections. For undefined terminology, the reader is referred to [2] and [3].

\section{The combinatorial Laplacian and spanning trees}

We consider a graph $G=(V, E)$ with vertex set $V=V(G)$ and edge set $E=E(G)$. Let $d_{v}$ denote the degree of $v$ in $G$. Here we assume $G$ contains no multiple edges. The combinatorial Laplacian $L$ of $G$ has rows and columns indexed by vertices of $G$, defined as follows.

$$
L(u, v)= \begin{cases}d_{v}-l_{v} & \text { if } u=v \\ -1 & \text { if } u \text { and } v \text { are adjacent } \\ 0 & \text { otherwise }\end{cases}
$$

where $l_{v}$ denotes the number of loops at $v$.

For a function $f: V \rightarrow \mathbb{R}$, we have

$$
L f(v)=\sum_{\substack{u \in V \\ u \sim v}}[f(v)-f(u)]
$$

One of the fundamental theorems in combinatorics is the matrix-tree theorem due to Kirchhoff [8] which states that the number of spanning trees in a graph is equal to the determinant of any principal submatrix of the combinatorial Laplacian.

For a graph $G$, the combinatorial Laplacian has non-negative eigenvalues, $0=$ $\rho_{0} \leq \rho_{1} \leq \ldots \rho_{n-1}$. The number of spanning trees, denoted by $\tau(G)$, can be related to the eigenvalues of $L$ as follows: (A proof can be found in [1]. For completeness, we briefly describe the proof here).

Theorem 1 For a graph $G$ on $n$ vertices, the number of spanning trees $\tau(G)$ is :

$$
\tau(G)=\frac{1}{n} \prod_{i \neq 0} \rho_{i}
$$


Proof: Suppose we consider the characteristic polynomial $p(x)$ of the combinatorial Laplacian $L$.

$$
p(x)=\operatorname{det}(L-x I)
$$

The coefficient of the linear term is exactly

$$
-\prod_{i \neq 0} \rho_{i}
$$

On the other hand, the coefficient of the linear term of $p(x)$ is -1 times the sum of the determinant of $n$ principal submatrix of $L$ obtained by deleting the $i$-th row and $i$-th column. By the matrix-tree theorem, the product $\prod_{i \neq 0} \rho_{i}$ is exactly $n$ times the number of spanning trees of $G$.

\section{The heat kernel and the zeta function}

In a graph $G$, let $S$ denote a finite connected induced subgraph of $G$. The combinatorial Laplacian $L_{S}$ restricted to $S$ is just

$$
L_{S} f(v)=\sum_{\substack{u \in S \\ u \sim v}}[f(v)-f(u)]
$$

for a function $f: S \rightarrow \mathbb{R}$ and a fixed $v \in S$.

Let $k$ denote the maximum degree of $G$. For $t \geq 0$, the heat kernel $h_{t}$ of an induced graph $S$ is defined by

$$
\begin{aligned}
h_{t} & =\sum_{i} e^{-\lambda_{i} t} P_{i} \\
& =e^{-t L_{S} / k} \\
& =I-\frac{t}{k} L_{S}+\frac{t^{2}}{2 ! k^{2}} L_{S}^{2}-\ldots
\end{aligned}
$$

where

$$
\lambda_{i}=\frac{\rho_{i}}{k}
$$

and $P_{i}$ denotes the projection into the eigenspace associated with eigenvalue $\rho_{i}$ of $L_{S}$. In particular, $h_{0}=I$, the identity matrix, and $h_{t}$ satisfies the heat equation

$$
\frac{d h_{t}}{d t}=-\frac{1}{k} L_{S} h_{t} .
$$


The trace formula in its most general form is

$$
\sum_{x} h_{t}(x, x)=\sum_{i} e^{-\lambda_{i} t}
$$

We define the trace function:

$$
\operatorname{Tr}\left(h_{t}\right)=\sum_{i} e^{-\lambda_{i} t}
$$

For a connected induced subgraph $S$, we consider the $\zeta$-function

$$
\zeta(s)=\sum_{i \neq 0} \frac{1}{\lambda_{i}^{s}}
$$

where $\lambda_{i}$ ranges over all nonzero eigenvalues of $\frac{1}{k} L_{S}$.

Therefore we have

$$
-\zeta^{\prime}(0)=\log \prod_{i \neq 0} \lambda_{i}
$$

where log denotes the natural logarithm.

Here we relate the number of spanning trees to the zeta function of $G$ (also see $[7])$.

Theorem 2 For a connected induced subgraph $S$ in a graph $G$ with maximum degree $k$, the number of spanning trees, denoted by $\tau(G)$, is equal to

$$
\tau(S)=\frac{k^{|S|-1}}{|S|} e^{-\zeta^{\prime}(0)} .
$$

Proof: Using (3) and Theorem 1, we have

$$
\begin{aligned}
\tau(S) & =\frac{1}{|S|} \prod_{i \neq 0} \rho_{i} \\
& =\frac{k^{|S|-1}}{|S|} \prod_{i \neq 0} \lambda_{i} \\
& =\frac{k^{|S|-1}}{|S|} e^{-\zeta^{\prime}(0)} .
\end{aligned}
$$


We consider

$$
\operatorname{Tr}^{*}\left(h_{t}\right)=\sum_{i \neq 0} e^{-\lambda_{i} t}
$$

Because of the fact that

$$
\int_{0}^{\infty} e^{-\lambda t} t^{z-1} d t=\frac{\Gamma(z)}{\lambda^{z}}
$$

we have the following:

\section{Theorem 3}

$$
\zeta(s)=\frac{1}{\Gamma(s)} \int_{0}^{\infty} t^{s-1} \operatorname{Tr}^{*}\left(h_{t}\right) d t
$$

We note that we also have the following Mellin inversion formula:

$$
\operatorname{Tr}^{*}\left(h_{t}\right)=\frac{1}{2 \pi i} \int t^{-s} \Gamma(s) \zeta(s) d s
$$

\section{The heat kernel for a path}

For the one-dimensional case, in an infinite path $P$, the vertices are labeled by integers and $x$ is adjacent to $x+1$ and $x-1$. The heat kernel of $P$ has been examined in [6] and here we state some facts that will be useful later.

The heat kernel $H_{t}$ of $P$ satisfies:

$$
\begin{aligned}
H_{t}(x, x) & =\lim _{n \rightarrow \infty} \frac{1}{n} \sum_{j=0}^{n-1} e^{-t \lambda_{j}} \\
& =\lim _{n \rightarrow \infty} \frac{1}{n} \sum_{j=0}^{n-1} e^{-t\left(1-\cos \frac{2 \pi j}{n}\right)} \\
& =\frac{2}{\pi} \int_{0}^{\pi / 2} e^{-2 t \sin ^{2} y} d y
\end{aligned}
$$

In general, the heat kernel $H_{t}(x, y)$ of an infinite path satisfies

$$
\begin{aligned}
H_{t}(x, x+a) & =\lim _{n \rightarrow \infty} \frac{1}{n} \sum_{j=0}^{n-1} e^{-t \lambda_{j}} e^{\frac{2 \pi i j a}{n}} \\
& =\frac{2}{\pi} \int_{0}^{\pi / 2} e^{-2 t \sin ^{2} y+2 i a y} d y \\
& =\frac{2}{\pi} \int_{0}^{\pi / 2} e^{-2 t \sin ^{2} y} \cos 2 a y d y
\end{aligned}
$$


We also need the following fact (see $[6])$ :

$$
\begin{aligned}
H_{t}(x, x+a) & =H_{t}(x, x-a) \\
& =(-1)^{a} e^{-t} \sum_{k \geq a} \frac{\left(\begin{array}{c}
2 k \\
k+a
\end{array}\right)}{k !}\left(\frac{-t}{2}\right)^{k} \\
& =e^{-t} \sum_{k \geq a} \frac{\left(\begin{array}{c}
a+2 k \\
k
\end{array}\right)}{k !}\left(\frac{t}{2}\right)^{a+2 k}
\end{aligned}
$$

The above equality can be used to show the following:

$$
\begin{aligned}
\sum_{a \in \mathbb{Z}^{+}} H_{t}(0,2 a+1) & =\frac{1}{2} \sum_{a \text { odd }} H_{t}(0,2 a+1) \\
& =\frac{1}{2} e^{-t} \sum_{k \text { odd }} \frac{(t)^{k}}{k !} \\
& =e^{-t} \frac{1}{4}\left(e^{t / 2}-e^{-t / 2}\right) \\
& =\frac{1-e^{-t}}{4}
\end{aligned}
$$

We will also use the following facts [2]:

$$
\begin{aligned}
\frac{d}{d t} H_{t}(x, y) & =-\frac{1}{2} L H_{t}(x, y) \\
& =-\frac{1}{2} \sum_{x^{\prime} \sim x}\left(H_{t}(x, y)-H_{t}\left(x^{\prime}, y\right)\right) .
\end{aligned}
$$

Also, we have

$$
H_{0}(x, y)= \begin{cases}1 & \text { if } x=y \\ 0 & \text { otherwise }\end{cases}
$$

\section{The heat kernel for lattice graphs}

In this section, we consider the $k$-dimensional lattice graphs and their induced subgraphs. We define the lattice graph $P_{n}^{(r)}$ to be the cartesian product of $k$ copies of an $n$-cycle. The infinite lattice graph $P^{(k)}$ is just by taking the limit of $P_{n}^{(r)}$ as $n$ approaches infinity. In particular, for the 2 -dimensional lattice graph $p^{(2)}$, each vertex is labelled by $(x, y), x, y \in \mathbb{Z}$. The vertex $(x, y)$ is adjacent to $(x+1, y),(x-$ $1, y),(x, y+1)$ and $(x, y-1)$. 
In an infinite 2 -dimensional lattice graph $P^{(2)}$, the heat kernel $H_{t}^{(2)}$ satisfies

$$
H_{t}^{(2)}((x, y),(x+a, y+b))=H_{t / 2}(x, x+a) H_{t / 2}(y, y+b)
$$

where $H_{t}$ is the heat kernel for an infinite path. In general, we have

$$
\left.H_{t}^{(r)}(x, x+a)\right)=\prod_{i=1}^{r} H_{t / r}\left(x_{i}, x_{i}+a_{i}\right)
$$

where $x=\left(x_{1}, \ldots, x_{r}\right)$.

For certain induced subgraphs of the $r$-dimensional lattice graphs $P^{(r)}$, we want to derive sharp estimates for the products of the nonzero eigenvalues of the combinatorial Laplacian. To do so, we consider the heat kernel $h_{t}$ of an induced subgraph $S$ of $P^{(r)}$. From (4), we have

$$
\begin{aligned}
\zeta(s) & =\frac{1}{\Gamma(s)} \int_{0}^{\infty} t^{s-1} \operatorname{Tr}^{*} h_{t} d t \\
& =\frac{1}{\Gamma(s)} \int_{0}^{\infty} t^{s-1}\left(\sum_{x \in S} h_{t}(x, x)-1\right) d t
\end{aligned}
$$

Of particular interest are subgraphs $S$ whose trace can be estimated by using the heat kernel of the lattice graph $P^{(r)}$. We define the function $\zeta_{0}$ as follows:

$$
\zeta_{0}(s)=\frac{1}{\Gamma(s)} \int_{0}^{\infty} t^{s-1} H_{t}^{(r)}(x, x) d t
$$

Using (5) and (11), we have

$$
\begin{aligned}
\zeta_{0}(s) & =\frac{1}{\Gamma(s)}\left(\frac{2}{\pi}\right)^{r} \int_{0}^{\pi / 2} \cdots \int_{0}^{\pi / 2} \int_{0}^{\infty} t^{s-1} e^{-2^{2-r}\left(\sin ^{2} x_{1}+\cdots+\sin ^{2} x_{r}\right)} d t d x_{1} \cdots d x_{r} \\
& =\left(\frac{2}{\pi}\right)^{r} \int_{0}^{\pi / 2} \cdots \int_{0}^{\pi / 2} \frac{1}{\left(2^{2-r}\left(\sin ^{2} x_{1}+\cdots+\sin ^{2} x_{r}\right)\right)^{s}} d x_{1} \cdots d x_{r}
\end{aligned}
$$

Therefore we have

$$
\zeta_{0}^{\prime}(0)=-\left(\frac{2}{\pi}\right)^{r} \int_{0}^{\pi / 2} \cdots \int_{0}^{\pi / 2} \log \left(2^{2-r}\left(\sin ^{2} x_{1}+\cdots+\sin ^{2} x_{r}\right)\right) d x_{1} \cdots d x_{r}(12)
$$

In particular, for the case of $r=2$, we have

$$
\begin{aligned}
\zeta_{0}^{\prime}(0) & =-\left(\frac{2}{\pi}\right)^{2} \int_{0}^{\pi / 2} \int_{0}^{\pi / 2} \log \left(\sin ^{2} x+\sin ^{2} y\right) d x d y \\
& =0.220050745 \cdots
\end{aligned}
$$


If $h_{t}$ can be approximated by $H_{t}^{(2)}$, then we can derive the following first-order estimate :

$$
\tau(S) \approx 4^{|S|-1} e^{-\zeta_{0}^{\prime}(0)|S|}
$$

This has a similar flavor as the formula of H. Weyl [10] for bounded regions of $\mathbb{R}^{k}$. To derive such estimates in a precise manner, we will examine the difference of $H_{t}$ and $h_{t}^{*}$ in the next section.

\section{The heat kernel of an induced subgraph of the lattice graph}

Suppose $S$ is an induced subgraph of the 2-dimensional lattice graph $P^{(2)}$. In order to enumerate the number of spanning trees of $S$, We will first establish the relation between the heat kernel of $S$ and the heat kernel of $P^{(2)}$.

We start with the combinatorical Laplacian $L_{S}$ for the induced subgraph $S$ which acts on functions $f: S \rightarrow \mathbb{R}$ as follows: For $x$ in $S$, we have

$$
L_{S} f(x)=\sum_{\substack{y \in S \\ y \sim x}}[f(x)-f(y)]
$$

In this section, we denote the heat kernel of $S$ by $h_{t}(x, y)=h(t, x, y)$ and the heat kernel of $P^{(r)}$ by $H_{t}^{(2)}(x, y)=\mathbf{H}(t, x, y)$. From the definition, we have

$$
\frac{d}{d t} h_{t}=-\frac{1}{4} L_{S} h_{t}, \quad \frac{d}{d t} \mathbf{H}_{t}=-\frac{1}{4} L \mathbf{H}_{t}
$$

We consider, for $x, y \in S$,

$$
\begin{aligned}
& \int_{0}^{t} \frac{d}{d s} \sum_{z \in S} h(t-s, x, z) \mathbf{H}(s, z, y) d s \\
= & \sum_{z \in S}[h(0, x, z) \cdot \mathbf{H}(t, z, y)-h(t, x, z) \cdot \mathbf{H}(0, z, y)] \\
= & \mathbf{H}(t, x, y)-h(t, x, y) .
\end{aligned}
$$


On the other hand, for fixed $x$ and $y$ in $S$, we have

$$
\begin{aligned}
& \mathbf{H}(t, x, y)-h(t, x, y) \\
= & \int_{0}^{t} \frac{d}{d s} \sum_{z \in S} h(t-s, x, z) \mathbf{H}(s, z, y) d s \\
= & \int_{0}^{t} \sum_{z \in S}\left(\frac{d}{d s} h(t-s, x, z) \cdot \mathbf{H}(s, z, y)+h(t-s, x, z) \cdot \frac{d}{d s} \mathbf{H}(s, z, y)\right] d s \\
= & \int_{0}^{t} \sum_{z \in S} \frac{1}{4}\left[L_{S} h(t-s, x, z) \cdot \mathbf{H}(s, z, y)-h(t-s, x, z) \cdot L \mathbf{H}(s, z, y)\right] d s \\
= & \int_{0}^{t} \frac{1}{4}\left[\sum_{\substack{z, w \in S \\
z \sim w}}\left(h(t-s, x, z)-h(t-s, x, w)(\mathbf{H}(s, z, y)-\mathbf{H}(s, w, y))-\sum_{z \in S} h(t-s, x, z) \cdot L \mathbf{H}(s, z, y)\right] d s\right. \\
= & -\frac{1}{4} \int_{0}^{t} \sum_{\substack{z \in S, z^{\prime} \notin S \\
z \sim z^{\prime}}} h(t-s, x, z)\left[\mathbf{H}(s, z, y)-\mathbf{H}\left(s, z^{\prime}, y\right)\right] d s
\end{aligned}
$$

Thus, we have

$$
h=\mathbf{H}+Q h
$$

where $Q$ is defined as follows:

$$
Q h(t, x, y)=\frac{1}{4} \int_{0}^{t} \sum_{\substack{z \in S, z^{\prime} \notin S \\ z \sim z^{\prime}}} h(t-s, x, z)\left[\mathbf{H}(s, z, y)-\mathbf{H}\left(s, z^{\prime}, y\right)\right] d s
$$

Thus we have

$$
h=\mathbf{H}+Q \mathbf{H}+Q^{2} \mathbf{H}+\ldots+Q^{r-1} \mathbf{H}+Q^{r} h .
$$

We have proved the following:

Theorem 4 For a connected induced subgraph $S$ in the 2-dimensional lattice graph, the heat kernel $h$ of $S$ satisfies the following:

$$
h=\mathbf{H}+Q \mathbf{H}+Q^{2} \mathbf{H}+\ldots+Q^{r-1} \mathbf{H}+Q^{r} h
$$

where

$$
Q h(t, x, y)=\frac{1}{4} \int_{0}^{t} \sum_{\substack{z \in S, z^{\prime} \notin S \\ z \sim z^{\prime}}} h(t-s, x, z)\left[\mathbf{H}(s, z, y)-\mathbf{H}\left(s, z^{\prime}, y\right)\right] d s
$$


As a consequence of Theorem 4, we also have the following useful fact:

Theorem 5 For a connected induced subgraph $S$ in the 2-dimensional lattice graph, the heat kernel $\mathbf{H}$ of the lattice graph satisfies the following:

$$
1-\sum_{x \in S} \mathbf{H}(t, x, y)=\frac{1}{4} \int_{0}^{t} \sum_{\substack{z \in S, z^{\prime} \notin S \\ z \sim z^{\prime}}}\left[\mathbf{H}(s, z, y)-\mathbf{H}\left(s, z^{\prime}, y\right)\right] d s
$$

Proof: The proof follows from Theorem 4 (for the case of $r=1$ ) and the fact that

$$
\sum_{x \in S} h(t, x, y)=1
$$

Using the definition of $Q,(14)$ can be written as

$$
1=\sum_{x \in S} \mathbf{H}(t, x, y)+Q 1(y)
$$

and

$$
1=\frac{1}{|S|} \sum_{x, y \in S}\left(\mathbf{H}(t, x, y)+Q \mathbf{H}(t, x, y)+\ldots+Q^{k} \mathbf{H}(t, x, y)\right)+Q^{k+1} 1
$$

where

$$
Q^{k} 1=\frac{1}{|S|} \sum_{y \in S} Q^{k} 1(y)
$$

\section{The heat kernel of a half plane}

In this section, we consider a special induced subgraph of the 2-dimensional lattice graph $P^{(2)}$. First, we consider a half plane $F$ which is an induced subgraph of $P^{(2)}$ with vertex set $\{v=(a, b): a \geq 0\}$.

We remark that from the definition of the combinatorial Laplacian in Section 2, we see that for a graph $G$ and a graph $G^{\prime}$ that is resulted by adding a loop to $G$, their combinatorial Laplacians are identical. An induced subgraph $S$ of a regular graph is not regular in general. We will often consider adding loops to vertices of $S$ which are adjacent to vertices not in $S$ so that all the degrees are equal in the 
resulting graph. The closure of an induced subgraph $S$, denoted by $\hat{S}$, is by adding $p$ loops to every vertex $v$ in $S$ which is incident to $p$ edges in the edge boundary $\partial S$. Clearly, the closure of an induced subgraphs have the same heat kernel.

Our goal is to express the heat kernel of a half plane in terms of the heat kernel of $P^{(2)}$.

Theorem 6 The heat kernel $\bar{h}$ of the halfplane $F$ satisfies, for vertices $u$ and $u$ of $F$,

$$
\bar{h}(t, u, v)=\mathbf{H}(t, u, v)+\mathbf{H}(t, u, \bar{v})
$$

where $\bar{v}$ is the mirrow image of the vertex $v$ with respect to the line $x=-1 / 2$.

Proof: We note that

$$
\bar{h}(t, u, v)=e^{-t} \sum_{r \geq 0} w_{r}(u, v) \frac{t^{r}}{r !}
$$

where $w_{r}(u, v)$ denote the number of walks of length $r$ from $u$ to $v$ in the closure the half plane F. It suffices to show that we can have $w_{r}(u, v)=w_{r}^{\prime}(u, v)+w{ }_{r}(u, \bar{v})$ where $w_{r}^{\prime}(u, v)$ is the number of walks of length $r$ from $u$ to $v$ in $P^{(2)}$ and $w^{\prime \prime}{ }_{r}(u, \bar{v})$ is the number of walks of length $r$ from $u$ to $\bar{v}$ in $P^{(2)}$.

We observe that a walk in the plane $P^{(2)}$ joining $u$ to $v$ corresponds to a walk in $\hat{F}$ by reflecting using the line $x=-1 / 2$. Namely, a walk which visits $\bar{v} \notin F$ shall be mapped to the corresponding walk which visit $v \in F$. Furthermore, an edge from $v$ which crosses the line $x=-1 / 2$ is corresponding to a loop at $v$. A walk of length $r$ from $u$ to $v$ in $P^{(2)}$ is then mapped to a walk of the same length from $v$ to $v$ in $\hat{F}$ with an even number of loops. Also, a walk of length $r$ from $u$ to $v$ in $\hat{F}$ which contains an odd number of loops is mapped to walks of length $r$ from $u$ to $\bar{v}$ in $P^{(2)}$. In fact, such correspondences give a bijection. Therefore, we have

$$
\bar{h}(t, u, v)=\mathbf{H}(t, u, v)+\mathbf{H}(t, u, \bar{v}) .
$$


We now define another operator $\mathbf{Q}$ as follows:

$$
\mathbf{Q} h(t, u, v)=\frac{1}{4} \int_{0}^{t} \sum_{\substack{z \in F, z^{\prime} \notin F \\ z \sim z^{\prime}}} h(t-s, u, z)\left[\mathbf{H}(s, z, v)-\mathbf{H}\left(s, z^{\prime}, v\right)\right] d s
$$

Theorem 7 For two vertices $x$ and $y$ in he half plane $F$, we have

$$
\mathbf{H}(t, u, \bar{v})=\mathbf{Q H}(t, u, v)+\mathbf{Q}^{2} \mathbf{H}(t, u, v)+\ldots
$$

where $\bar{v}$ is the mirrow image of the vertex $v$ with respect to the line $x=-1 / 2$.

Proof: The proof follows from Theorem 6, which says

$$
\bar{h}(t, x, y)-\mathbf{H}(t, x, y)=\mathbf{H}(t, x, \bar{y}) .
$$

Then we use Theorem 4 which states that

$$
\bar{h}(t, x, y)-\mathbf{H}(t, x, y)=\mathbf{Q H}(t, x, y)+\mathbf{Q}^{2} \mathbf{H}(t, x, y)+\ldots .
$$

Another consequence of $(15)$ is the following:

\section{Theorem 8}

$$
\sum_{\substack{a \in \mathbb{Z}^{+} \\ v=(a, 0)}} \mathbf{Q} f(t, v, v)=\frac{1}{4} \int_{0}^{t} \sum_{x \in F} f\left(t-s, x, z_{0}\right)\left[\mathbf{H}\left(s, z_{0}, v\right)-\mathbf{H}\left(s, z_{0}^{\prime}, x\right)\right] d s
$$

for $z_{0}=(0,0), z_{0}^{\prime}=(-1,0)$ and for any $f$.

Proof: We sum (15) over all vertices $v$ in $F$.

$$
\begin{aligned}
\sum_{\substack{a \in \mathbb{Z}^{+} \\
v=(a, 0)}} \mathbf{Q} f(t, v, v) & =\frac{1}{4} \int_{0}^{t} \sum_{\substack{a \in \mathbb{Z}^{+} \\
v=(a, 0)}} \sum_{\substack{z \in F, z^{\prime} \notin F \\
z \sim z^{\prime}}} f(t-s, v, z)\left[\mathbf{H}(s, z, v)-\mathbf{H}\left(s, z^{\prime}, v\right)\right] d s \\
& =\frac{1}{4} \int_{0}^{t} \sum_{x \in F} f(t-s, x, z)\left[\mathbf{H}\left(s, z_{0}, v\right)-\mathbf{H}\left(s, z_{0}^{\prime}, x\right)\right] d s
\end{aligned}
$$




\section{Bounding the number of spanning trees}

Suppose $S$ is an induced subgraph of the 2-dimensional lattice graph $P^{(2)}$. We will prove the main theorem.

Theorem 9 For a connected induced subgraph $S$ in the 2-dimensional lattice graph, the number of spanning trees $\tau(S)$ of $S$ satisfies:

$$
\frac{1}{|S|}\left(4 e^{-\alpha}\right)^{|S|-1} e^{-\beta|\partial S|(1-1 /|S|)} \leq \tau(S) \leq \frac{1}{|S|} 4^{|S|-1} e^{-\alpha\left(|S|-|\partial S|^{2} /|S|\right)}
$$

where

$$
\begin{aligned}
\alpha & =-\left(\frac{2}{\pi}\right)^{2} \int_{0}^{\pi / 2} \int_{0}^{\pi / 2} \log \left(\left(\sin ^{2} x_{1}+\sin ^{2} x_{2}\right)\right) d x_{1} d x_{2} \\
& \approx .2200507 \ldots \\
\beta & =-\frac{1}{2 \pi} \int_{0}^{\pi / 2}\left(\log \sin ^{2} x-\log \left(1+\sin ^{2} x\right)\right) d x \\
& \approx .44068679 \ldots
\end{aligned}
$$

Proof: From Theorem 4, we have

$$
h=\mathbf{H}+Q \mathbf{H}+Q^{2} \mathbf{H}+\ldots+Q^{r-1} \mathbf{H}+Q^{r} h .
$$

We consider the trace of $h$. For simplicity, we write

$$
\operatorname{Tr}_{S} \mathbf{H}_{t}=\sum_{x \in S} Q \mathbf{H}(t, x, x) .
$$

We have

$$
\begin{aligned}
\operatorname{Tr}_{S} Q \mathbf{H}_{t} & =\sum_{x \in S} Q \mathbf{H}_{t}(x, x) \\
& =\sum_{\substack{z \in S, z^{\prime} \notin S \\
z \sim z^{\prime}}} \frac{1}{4} \sum_{x \in S} \int_{0}^{t} \mathbf{H}(t-s, x, z)\left[\mathbf{H}(s, z, x)-\mathbf{H}\left(s, z^{\prime}, x\right)\right] d s \\
& \leq \sum_{\substack{z \in S, z^{\prime} \notin S \\
z \sim z^{\prime}}} \frac{1}{4} \sum_{x \in F_{z}} \int_{0}^{t} \mathbf{H}(t-s, x, z)\left[\mathbf{H}(s, z, x)-\mathbf{H}\left(s, z^{\prime}, x\right)\right] d s
\end{aligned}
$$

where $F_{z}$ is the half plane consisting of all points closer to $z$ than to $z^{\prime}$. Thus, we have

$$
\operatorname{Tr}_{S} Q \mathbf{H}_{t} \leq \frac{|\partial S|}{4} \sum_{x \in F_{z}} \int_{0}^{t} \mathbf{H}(t-s, x, z)\left[\mathbf{H}(s, z, x)-\mathbf{H}\left(s, z^{\prime}, x\right)\right] d s
$$


Using Theorem 8, we get

$$
\begin{aligned}
\operatorname{Tr}_{S} Q \mathbf{H}_{t} & =\sum_{x \in S} Q \mathbf{H}(t, x, x) \\
& \leq|\partial S| \sum_{\substack{a \in \mathbb{Z}^{+} \\
v=(a, 0)}} \mathbf{Q H}(t, v, v)
\end{aligned}
$$

By repeatedly using Theorem 8 , we have

$$
\begin{aligned}
\operatorname{Tr}_{S} Q^{j} \mathbf{H}_{t} & =\sum_{x \in S} Q^{j} \mathbf{H}(t, x, x) \\
& \leq|\partial S| \sum_{\substack{a \in \mathbb{Z}^{+} \\
v=(a, 0)}} \mathbf{Q}^{j} \mathbf{H}(t, v, v)
\end{aligned}
$$

Summing over $j$, we have

$$
\begin{aligned}
\sum_{j \geq 1} \operatorname{Tr}_{S} Q^{j} \mathbf{H}_{t} & \leq|\partial S| \sum_{\substack{a \in \mathbb{Z}^{+} \\
v=(a, 0)}} \sum_{j \geq 1} \mathbf{Q}^{j} \mathbf{H}(t, v, v) \\
& =|\partial S| \sum_{\substack{a \in \mathbb{Z}^{+} \\
v=(a, 0)}} \mathbf{H}(t, v, \bar{v})
\end{aligned}
$$

by using Theorem 7 .

Then we have

$$
\begin{aligned}
\zeta(s)= & \frac{1}{\Gamma(s)} \int_{0}^{\infty} t^{s-1}\left(\operatorname{Tr}\left(h_{t}\right)-1\right) d t \\
= & \frac{|S|}{\Gamma(s)} \int_{0}^{\infty} t^{s-1} \mathbf{H}(t, x, x) d t+\frac{1}{\Gamma(s)} \int_{0}^{\infty} t^{s-1}\left(\operatorname{Tr}_{S} Q \mathbf{H}_{t}+\ldots+\operatorname{Tr} Q^{r} \mathbf{H}_{t}\right) \\
& -\frac{1}{\Gamma(s)} \int_{0}^{\infty} t^{s-1}\left(1-Q^{r+1} 1\right) d t \\
= & \zeta_{0}(s)|S|+\zeta_{1}(s)+\zeta_{2}(s)
\end{aligned}
$$

From the previous section, we know that

$$
\begin{aligned}
\zeta_{0}^{\prime}(0) & =-\left(\frac{2}{\pi}\right)^{2} \int_{0}^{\pi / 2} \int_{0}^{\pi / 2} \log \left(\sin ^{2} x+\sin ^{2} y\right) d x d y \\
& =0.220050745 \cdots
\end{aligned}
$$

It suffices to estimate and bound $\zeta_{1}^{\prime}(0)$ and $\zeta_{2}^{\prime}(0)$. We will use the fact that for differentiable functions $f, g$ with $f(0)=g(0)$, if $f(x) \leq g(x)$ for $x \geq 0$, then $f^{\prime}(0) \geq g^{\prime}(0)$. 


$$
\begin{aligned}
\zeta_{1}(s) & \leq \frac{1}{\Gamma(s)} \int_{0}^{\infty} t^{s-1}\left(\operatorname{Tr}_{S} Q \mathbf{H}_{t}+\ldots\right) d t \\
& \leq \frac{|\partial S|}{\Gamma(s)} \int_{0}^{\infty} t^{s-1} \sum_{\substack{a \in \mathbb{Z}^{+} \\
v=(a, 0)}} \mathbf{H}(t, v, \bar{v}) d t \\
& =\frac{|\partial S|}{4 \Gamma(s)} \int_{0}^{\infty} t^{s-1}\left(1-e^{-t}\right) H(t / 2,0,0) d t
\end{aligned}
$$

by using (7). Therefore we have

$$
\begin{aligned}
\zeta_{1}(s) & \leq \frac{|\partial S|}{4 \Gamma(s)} \int_{0}^{\infty} t^{s-1}\left(1-e^{-t}\right) \frac{2}{\pi} \int_{0}^{\pi / 2} e^{-t \sin ^{2} x} d x d t \\
& =\frac{|\partial S|}{2 \pi} \int_{0}^{\pi / 2}\left(\frac{1}{\sin ^{2 s} x}-\frac{1}{\left(1+\sin ^{2} x\right)^{s}}\right) d x
\end{aligned}
$$

Consequently,

$$
\begin{aligned}
\zeta_{1}^{\prime}(0) & \leq-\frac{|\partial S|}{2 \pi} \int_{0}^{\pi / 2}\left(\log \sin ^{2} x-\log \left(1+\sin ^{2} x\right)\right) d x \\
& =\beta|\partial S| \\
& \approx .44068679 \ldots|\partial S|
\end{aligned}
$$

It remains to bound $\zeta_{2}$. From Theorem 4 , we see that for any $x \in S$,

$$
1-Q^{r+1} 1(x)=\sum_{y \in S} \mathbf{H}(x, y)+\sum_{j=1}^{r} \sum_{y \in S} Q^{j} \mathbf{H}(t, x, y) .
$$

We also note that for $x \neq y$,

$$
\lim _{s \rightarrow 0} \frac{1}{\Gamma(s)} \int_{0}^{\infty} t^{s-1} \mathbf{H}(t, x, y) d t=0
$$

In fact, we have, in general,

$$
\lim _{s \rightarrow 0} \frac{1}{\Gamma(s)} \int_{0}^{\infty} t^{s-1} Q^{j} \mathbf{H}(t, x, y) d t=0
$$

Therefore we have

$$
\lim _{s \rightarrow 0} \zeta_{2}(0)=\frac{1}{n}\left(\zeta_{0}(0)+\zeta_{1}(0)\right)
$$


Clearly, we have

$$
\begin{aligned}
\zeta_{2}(x) & \leq-\frac{1}{\Gamma(s)} \int_{0}^{\infty} \sum_{j \geq 0} \sum_{x, y} \mathbf{H}(t, x, y) d t \\
& \leq-\frac{1}{|S|}\left(\zeta_{0}(0)+\zeta_{1}(0)\right)-\frac{1}{\Gamma(s)} \int_{0}^{\infty} \sum_{j \geq 0} \sum_{x \neq y} \mathbf{H}(t, x, y) d t \\
& \leq-\frac{1}{|S|}\left(\zeta_{0}(0)|S|+\zeta_{1}(0)\right) .
\end{aligned}
$$

Altogether, we have

$$
\zeta^{\prime}(0) \leq \alpha(|S|-1)+\beta|\partial S|\left(1-\frac{1}{|S|}\right)
$$

This implies

$$
\tau(S) \geq \frac{1}{|S|}\left(4 e^{-\alpha}\right)^{|S|-1} e^{-\beta|\partial S|(1-1 /|S|)} .
$$

To upper bound $\tau(S)$, it is enough to lower bound $\zeta^{\prime}(0)$. We consider

$$
\begin{aligned}
\zeta(s)= & \frac{1}{\Gamma(s)} \int_{0}^{\infty} t^{s-1}\left(\operatorname{Tr}\left(h_{t}\right)-1\right) d t \\
\geq & \frac{1}{\Gamma(s)} \int_{0}^{\infty} t^{s-1}\left(\sum_{x \in S} \mathbf{H}(t, x, x)-\frac{1}{|S|} \sum_{x, y \in S} \mathbf{H}(t, x, y)\right) d t \\
& +\frac{1}{\Gamma(s)} \int_{0}^{\infty} t^{s-1}\left(\sum_{r \geq 1} \sum_{x \in S} Q^{r} \mathbf{H}(t, x, x)-\frac{1}{|S|} \sum_{x, y \in S} Q^{r} \mathbf{H}(t, x, y)\right) d t \\
\geq & \frac{1}{\Gamma(s)} \int_{0}^{\infty} t^{s-1} \sum_{x \in S} \mathbf{H}(t, x, x)-\frac{1}{|S|} \sum_{x, y \in S} \mathbf{H}(t, x, y) d t \\
= & |S| \zeta_{0}(s)+\zeta_{3}(s)
\end{aligned}
$$

where

$$
\zeta_{3}(s)=-\frac{1}{\Gamma(s)} \int_{0}^{\infty} t^{s-1} \frac{1}{|S|} \sum_{x, y \in S} \mathbf{H}(t, x, y) d t
$$

We consider

$$
F(t)=\sum_{x, y \in S} \mathbf{H}(t, x, y)-|\partial S|^{2} \mathbf{H}(t, x, x)
$$

We note that

$$
\begin{aligned}
\frac{d}{d t} F(t) & =-\sum_{x \in S} \sum_{y \in S} \frac{1}{4} L H(t, x, y)+\frac{|\partial S|^{2}}{4} L \mathbf{H}(t, x, x) \\
& =-\frac{1}{4} \sum_{x \in S} \sum_{\substack{y \in S, y^{\prime} \notin S \\
y \sim y^{\prime}}}\left(\mathbf{H}(t, x, y)-\mathbf{H}\left(t, x, y^{\prime}\right)\right)+\frac{|\partial S|^{2}}{4} L \mathbf{H}(t, x, x)
\end{aligned}
$$


and

$$
\begin{aligned}
\frac{d^{2}}{d t^{2}} F(t)= & -\frac{1}{16} \sum_{\substack{x \in S, x^{\prime} \notin S \\
x \sim x^{\prime}}} \sum_{\substack{y \in S, y^{\prime} \notin S \\
y \sim y^{\prime}}}\left(\mathbf{H}(t, x, y)-\mathbf{H}\left(t, x, y^{\prime}\right)-\left(\mathbf{H}\left(t, x^{\prime}, y\right)-\mathbf{H}\left(t, x^{\prime}, y^{\prime}\right)\right)\right. \\
& -\frac{|\partial S|^{2}}{16} L^{2} \mathbf{H}(t, x, x) \\
\leq & 0
\end{aligned}
$$

Therefore, we have

$$
\frac{d}{d t} F(t) \leq \lim _{t \rightarrow \infty} \frac{d}{d t} F(t)=0 .
$$

Consequently,

$$
F(t) \leq \lim _{t \rightarrow \infty} F(t)=0
$$

and

$$
\sum_{x, y \in S} \mathbf{H}(t, x, y) d t \leq|\partial S|^{2} \mathbf{H}(t, x, x) .
$$

This implies

$$
\begin{aligned}
\zeta_{3}(s) & =-\frac{1}{\Gamma(s)} \int_{0}^{\infty} t^{s-1} \frac{1}{|S|} \sum_{x, y \in S} \mathbf{H}(t, x, y) d t \\
& \geq-\frac{1}{\Gamma(s)} \int_{0}^{\infty} t^{s-1} \frac{|\partial S|^{2}}{|S|} \mathbf{H}(t, x, x) d t \\
& \geq-\frac{1}{\Gamma(s)} \int_{0}^{\infty} t^{s-1} \frac{|\partial S|^{2}}{|S|} \mathbf{H}(t, x, x) d t
\end{aligned}
$$

Therefore we have

$$
\zeta_{3}(s) \geq=-\frac{1}{\Gamma(s)} \int_{0}^{\infty} t^{s-1} \frac{|\partial S|^{2}}{|S|} \mathbf{H}(t, x, x) d x
$$

and

$$
\zeta_{3}^{\prime}(0) \geq-\frac{|\partial S|^{2}}{|S|} \alpha
$$

Therefore

$$
\tau(S) \leq \frac{1}{|S|} 4^{|S|-1} e^{-\alpha\left(|S|-|\partial S|^{2} /|S|\right)} .
$$

This completes the proof of Theorem 3 . 
We remark that the various constants in Theorem 9 can be improved by imposing additional conditions on the induced subgraph $S$. For example, suppose that the induced subgraph $S$ consists of vertices within an area with boundary consisting of horizontal and vertical line segments. If the boundary line segments are large, we can derive sharper estimates for $\zeta^{\prime}(0)$. In additional, various convexity conditions $[4,5]$ can be explored. However, these further considerations will not be included here.

\section{Acknowledgement}

I wish to thank John Schotland for first introducing me to many aspects of the zeta function for graphs. I am grateful to Professor Robert Langlands for numerous discussion and particularly for the computational data on spanning trees of rectangular lattices. Special thanks are due to Professor S. T. Yau for illuminating discussions on various powerful techniques using the heat kernels.

\section{References}

[1] N.L. Biggs, Algebraic Graph Theory, (2nd ed.), Cambridge University Press, Cambridge, 1993.

[2] F. R. K. Chung, Spectral Graph Theory, CBMS Lecture Notes, 1997, AMS Publication.

[3] F. R. K. Chung and Robert P. Langlands, A combinatorial Laplacian with vertex weights, J. Comb. Theory, (A), 75 (1996), 316-327.

[4] F. R. K. Chung and S. -T. Yau, Eigenvalue inequalities of graphs and convex subgraphs, Communications on Analysis and Geometry, 5 (1998), 575-624.

[5] F. R. K. Chung and S. -T. Yau, A Harnack inequality for homogeneous graphs and subgraphs, Communications on Analysis and Geometry, 2 (1994), 628-639.

[6] F. R. K. Chung and S. -T. Yau, A combinatorial trace formula, Tsing Hua Lectures on Geometry and Analysis, (ed. S.-T. Yau), International Press, Cambridge, Massachusetts, 1997, 107-116. 
[7] F. R. K. Chung and S. -T. Yau, Coverings, heat kernels and spanning trees, Electronic Journal of Combinatorics6 (1999), \#R12.

[8] F. Kirchhoff, Über die Auflösung der Gleichungen, auf welche man bei der Untersuchung der linearen Verteilung galvanischer Ströme geführt wird, Ann. Phys. Chem. 72 (1847), 497-508.

[9] H. P. McKean, Jr. and I. M. Singer, Curvature and the eigenvalues of the Laplcian, J. Differential Geometry 1 (1967), 43-69.

[10] H. Weyl, Das asymptotische Verteilungsgesetz der Eigenschwingungen eines beliebig gestalteten elastischen K oopers, Rend. Cir. Mat. Palermo 39 (1950), 1-50. 